\title{
CROSSED PRODUCTS OF SIMPLE RINGS
}

\author{
NOBUO NOBUSAWA
}

Let $A$ be the algebra of all $q \times q$ matrices over a division ring $D$. Suppose there is given a group $G$ of $n$ automorphisms of $A$ such that

(i) the fixed subring $S$ of $G$ is a simple ring such that $[A: S]=n$ and

(ii) $G A_{r}$ coincides with the totality of all homomorphisms of the $S$-left module $A$ to itself where $A_{r}$ is the ring of right multiplication of elements of $A$.

Suppose also that there is given a factor system $\left\{c_{\sigma, \tau}\right\}(\sigma, \tau \in G)$ in the center $K$ of $A$. Then a crossed product of $A$ and $G$ is defined via the same formulae as in the commutative case. (See [2].) The purpose of this note is to investigate the splitting property of a factor system by an extension of $S$ as well as of $A$. This is a generalization of a wellknown theorem for the commutative case as well as of a result given in [2].

To begin with, we shall consider a purely transcendental extension of $A$ as follows. Let $x_{1}, \cdots, x_{m}$ be $m$ variables. Let $D\left[x_{1}, \cdots, x_{m}\right]$ be the polynomial ring of $x_{1}, \cdots, x_{m}$ over $D$. We suppose $x_{i}$ lie in the center of the ring. Then the quotient division ring of $D\left[x_{1}, \cdots, x_{m}\right]$ is denoted by $D\left(x_{1}, \cdots, x_{m}\right)$. The existence of the quotient ring is clear from a general theory of quotient ring, or it can be proved directly as follows. Generally let $\Gamma$ be a ring with no divisor of zero. Moreover suppose that for any nonzero elements $a$ and $b$ of $\Gamma$ there exist nonzero elements $a^{\prime}, b^{\prime}, a^{\prime \prime}$ and $b^{\prime \prime}$ such that $a a^{\prime}=b b^{\prime}$ and $a^{\prime \prime} a=b^{\prime \prime} b$. We consider the set of formal elements $a^{-1} b$ and $c d^{-1}$ $\left(a, b, c, d \in \Gamma\right.$ and $a \neq 0, d \neq 0$ ). Define $a_{1}^{-1} b_{1}=a_{2}^{-1} b_{2}$ if and only if there exist nonzero elements $c$ and $d$ such that $a_{1} c=b_{1} d$ and $a_{2} c=b_{2} d$. It is a good exercise to show that the above equivalent relation is well defined. Similarly, define $b_{1} a_{1}^{-1}=b_{2} a_{2}^{-1}$ if and only if there exist nonzero elements $c$ and $d$ such that $c a_{1}=d b_{1}$ and $c a_{2}=d b_{2}$. Also define $a^{-1} b=c d^{-1}$ if and only if $a c=b d$. To verify that this is well defined is also a good exercise. How to define the algebraic operations in this set is now almost clear. For example, $\left(a^{-1} b\right)\left(c^{-1} d\right)=\left(c^{\prime} a\right)^{-1} b^{\prime} d$, where $b c^{-1}=c^{-1} b^{\prime}$. Also, $a^{-1} b+c^{-1} d=\left(c^{\prime \prime} a\right)^{-1}\left(c^{\prime \prime} b+a^{\prime \prime} d\right)$, where $a c^{-1}=c^{\prime \prime-1} a^{\prime \prime}$. The set is a division ring called a quotient ring of $\Gamma$ and it is uniquely determined up to isomorphism. To apply this general theory to our case, we must verify that the above mentioned conditions of $\Gamma$ are satisfied for $D\left[x_{1}, \cdots, x_{m}\right]$. To see it, take $D\left[x_{1}\right]$ first. The above

Received by the editors September 10, 1968 and, in revised form, April 14, 1969. 
conditions of $\Gamma$ are easy consequences of the division algorithm in $D\left[x_{1}\right]$. The general case can be proved easily by induction. Thus $D\left(x_{1}, \cdots, x_{m}\right)$ is constructed. Then we let $A\left(x_{1}, \cdots, x_{m}\right)$ be the algebra of all $q \times q$ matrices over $D\left(x_{1}, \cdots, x_{m}\right)$.

The next step is to extend $G$ to an automorphism group of $A\left(x_{1}, \cdots, x_{m}\right)$. We set $m=n-1$ and let $G=\left\{\sigma_{0}=\epsilon, \sigma_{1}, \cdots, \sigma_{m}\right\}$. ( $\epsilon$ is the identity.) We rather denote $x_{i}$ by $x_{\sigma}\left(\sigma=\sigma_{i}\right)$ and set $x_{\epsilon}=1$. Now define

$$
x_{\sigma}^{\tau}=x_{\tau}^{-1} x_{\sigma \tau} c_{\sigma, \tau}
$$

for a given factor system $\left\{c_{\sigma, \tau}\right\}$, where we may assume without losing generality that $c_{\sigma, \epsilon}=c_{\epsilon, \sigma}=1$. This is of course one of classical devices in the theory of factor sets. Using the identities $c_{\tau, \rho}^{-1} c_{\sigma \tau, \rho} c_{\sigma, \tau}^{\rho}=c_{\sigma, \tau \rho}$, we can prove that $\left(x_{\sigma}^{\tau}\right)^{\rho}=x_{\sigma}^{\tau \rho}$. Operating $\tau$ on elements of $A$ as usual, we get automorphisms of $A\left(x_{1}, \cdots, x_{m}\right)$. (The mappings $\tau$ are first defined on $D\left[x_{1}, \cdots, x_{m}\right]$, and then we generalize them on $D\left(x_{1}\right.$, $\left.\cdots, x_{m}\right)$ by setting $\left(a^{-1}\right)^{\tau}=\left(a^{r}\right)^{-1}$ for $a$ in $D\left[x_{1}, \cdots, x_{m}\right]$.) Thus $G$ is considered to be an automorphism group of $A\left(x_{1}, \cdots, x_{m}\right)$. Moreover, what is more important, $G$ is a group of outer automorphisms of $A\left(x_{1}, \cdots, x_{m}\right)$ since every element except the identity of $G$ acts nontrivially on the center $K\left(x_{1}, \cdots, x_{m}\right)$ of the ring.

Now we are at the position to apply an elementary part of (outer) Galois theory of simple rings. (See [1].) If we denote the fixed subring of $G$ by $B, B$ is a simple ring and $\left[A\left(x_{1}, \cdots, x_{m}\right): B\right]=n[1$, Theorem 1, p. 282]. Moreover, if we denote by $u_{1}, \cdots, u_{n}$ a basis of the $S$-left module $A$, then it is also a basis of the $B$-left module $A\left(x_{1} \cdots, x_{m}\right)$. The main object of constructing the Galois extension $A\left(x_{1}, \cdots, x_{m}\right) / B$ was in that the factor system $\left\{c_{\sigma, \tau}\right\}$ splits in it as is seen from (1). However, the above result is still not satisfactory because $B$ is too general. What we wish to get is a Galois extension $A^{\prime} / B^{\prime}$ with $B^{\prime}$ in $A$ in which $\left\{c_{\sigma, \tau}\right\}$ splits. The natural way to get such $B^{\prime}$ is a specialization method in a sense of algebraic geometry.

A brief discussion of specialization is as follows. Let $R\{t\}$ be a ring of all formal power series for a ring $R$ and a variable $t$, i.e., elements of $R\{t\}$ are $\sum_{i=-\infty}^{\infty} r_{i} t^{i}$ where almost all $r_{i}$ are supposed to be 0 for negative integers $i$. We define a mapping of $R\{t\}$ to $R$ and a symbol $\infty$; map $\sum r_{i} t^{i}$ to $r_{0}$ if all $r_{i}=0$ for negative $i$, and to $\infty$ otherwise. Returning to $D\left(x_{1}, \cdots, x_{m}\right)$, set $t_{i}=x_{i}-1(i=1, \cdots, m)$, and therefore $D\left(x_{1}, \cdots, x_{m}\right)=D\left(t_{1}, \cdots, t_{m}\right)$. Then embed $D\left(t_{1}, \cdots, t_{m}\right)$ into the formal power series (division) ring $D\left\{t_{1}\right\} \cdots\left\{t_{m}\right\}$ in a natural way. (First embed $D\left(t_{1}\right)$ into $D\left\{t_{1}\right\}$, then $D\left(t_{1}, t_{2}\right)$ into $D\left\{t_{1}\right\}\left\{t_{2}\right\}$, and so on.) On the other hand, we can generalize the above mentioned 
specialization to a case of several variables as follows. First, apply the above process to $R\left\{t_{m}\right\}$, where $R=D\left\{t_{1}\right\} \cdots\left\{t_{m-1}\right\}$. Next, map $R=R^{\prime}\left\{t_{m-1}\right\}$ to $R^{\prime}$ and $\infty$, where $R^{\prime}=D\left\{t_{1}\right\} \cdots\left\{t_{m-2}\right\}$. Continuing this process in this order, we get a mapping of $D\left\{t_{1}\right\} \cdots\left\{t_{m}\right\}$ to $D$ and $\infty$. ( $\infty$ is always mapped to $\infty$.) The mapping is called a specialization induced by $x_{m} \rightarrow 1, x_{m-1} \rightarrow 1, \cdots$, and $x_{1} \rightarrow 1$ (in this order). Restrict the mapping to the subset $D\left(x_{1}, \cdots, x_{m}\right)$, we get a specialization of it. To extend it to $A\left(x_{1}, \cdots, x_{m}\right)$, simply map each entry of a matrix to get a matrix whose entries are either elements of $D$ or $\infty$. If $\infty$ appears in a result, we set the result $\infty$.

Now let $B^{\prime}$ be the finite image of $B$ in the specialization. Clearly $S \subseteq B^{\prime} \subseteq A$. Denote the totality of elements of $B$ that have finite images in the specialization by $V(B)$, and the totality of elements of $B$ that are mapped to 0 by $P(B)$. Set

$$
U=\left\{\sum_{i} b_{i} u_{i} \mid b_{i} \in V(B), i=1, \cdots, n\right\}
$$

and

$$
P=\left\{\sum_{i} p_{i} u_{i} \mid p_{i} \in P(B), i=1, \cdots, n\right\}
$$

Lemma. $U$ is a ring and $P$ is an ideal of $U$.

Proof. To prove lemma, it is sufficient to show that $u_{i} b \in U$ and $u_{i} p \in P$, where $b \in V(B)$ and $p \in P(B)$. We need some preparation. Observing the condition (ii) of $A$, we see that every $S$-left homomorphism $\phi$ of $A$ to $S$ is expressed as $\phi=\sum a_{i r} \sigma_{i}\left(a_{i} \in A\right)$ and $(a \phi)^{\sigma}=a \phi$ for every $\sigma$ in $G$. Therefore, $a_{1}=a_{2}=\cdots=a_{n}$, or $\phi=a_{r}\left(\sum \sigma_{i}\right)$ with an element $a$. Especially $S$-left homomorphisms which map $u_{j}$ to 1 and $u_{i}(i \neq j)$ to 0 are expressed as $v_{j r}\left(\sum \sigma_{k}\right)$ with some elements $v_{j}$. This implies $\operatorname{Tr}_{G}\left(u_{i} v_{j}\right)=\delta_{i j}$. Returning to the proof of lemma, express $u_{i} b=\sum b_{k} u_{k}$ with $b_{k}$ in $B$. Then $\operatorname{Tr}_{G}\left(u_{i} b v_{j}\right)=b_{j}$. But $\operatorname{Tr}_{G}\left(u_{i} b v_{j}\right) \in V(B)$ if $b \in V(B)$. This shows that $u_{i} b$ is contained in $U$. A similar discussion shows that $u_{i} p \in P$, which concludes the proof.

Lastly, we can show that $x_{\sigma}$ are in $U$ but not in $P$. For, set $x_{\sigma}$ $=\sum b_{i} u_{i}$. As in above, $b_{i}=\operatorname{Tr}_{G}\left(x_{\sigma} v_{i}\right)$, the latter being in $V(B)$ since $x_{\sigma}^{\tau} v_{i}^{\tau}$ are mapped to $c_{\sigma, \tau} v_{i}^{\tau}$, namely, have finite images. Thus $x_{\sigma} \in U$. Clearly $x_{\sigma}$ are not in $P$. Now, we consider the residue class ring $A^{\prime}$ $=U / P$. Since $G$ induces an automorphism group of $U$ as well as that of $P$, it induces an automorphism group of $A^{\prime}$. The fixed subring of $G$ in $U / P$ is identified with $B^{\prime}$. Moreover, if we denote the residue 
classes represented by $x_{\sigma}$ by $x_{\sigma}^{\prime}$, then $x_{\sigma}^{\prime \tau}=x_{\tau}^{\prime-1} x_{\sigma \tau}^{\prime} c_{\sigma, \tau}$ in $A^{\prime}$. Thus we have obtained a final result.

THEOREM. The factor system $\left\{c_{\sigma, \tau}\right\}$ splits in $A^{\prime} / B^{\prime}$.

\section{REFERENCES}

1. T. Nakayama, Galois theory of simple rings, Trans. Amer. Math. Soc. 73 (1952), 276-292. MR 14, 240.

2. N. Nobusawa, On crossed products of division rings, Nagoya Math. J. 35 (1969), 47-51.

UNIVERSITY OF HAWAII 